\title{
CONTAMINACIÓN POR METALES PESADOS: IMPLICACIONES EN SALUD, AMBIENTE Y SEGURIDAD ALIMENTARIA
}

\section{Heavy metals contamination: implications for health and food safety}

\author{
Yulieth C. Reyes ${ }^{1}$, Inés Vergara², Omar E. Torres ${ }^{1}$ Mercedes Díaz $^{2}$, Edgar E. González ${ }^{1}$ \\ ${ }^{1}$ Pontificia Universidad Javeriana (Bogotá D.C., Colombia), Facultad de Ingeniería, Instituto Geofísico, \\ egonzale@javeriana.edu.co \\ ${ }^{2}$ Universidad Pedagógica y Tecnológica de Colombia. Universidad Pedagógica y Tecnológica de Colombia, (Sogamoso- \\ Boyacá, Colombia). Facultad de Ingeniería Geológica, Seccional Sogamoso, \\ mercedes.diaz@uptc.edu.co.
}

(Recibido mayo 2 de 2016 y aceptado junio 13 de 2016)

\begin{abstract}
Resumen
La contaminación por metales pesados y metaloides en recursos hídricos, suelos y aire plantea una de las más severas problemáticas que comprometen la seguridad alimentaria y salud pública a nivel global y local. En esta revisión, se aborda el problema específico de contaminación por mercurio $(\mathrm{Hg})$, Arsénico $(\mathrm{As})$, Cadmio $(\mathrm{Cd})$ y Plomo $(\mathrm{Pb})$ en ambiente y alimentos. Se presenta una descripción sobre las fuentes de contaminación y exposición en seres vivos así como la incorporación y retención en alimentos y productos de consumo humano. Se abordan casos de estudio y resultados obtenidos en algunos países del mundo incluido Colombia.
\end{abstract}

Palabras clave: Metales pesados, Metaloides, Mercurio, Arsénico, Cadmio, Plomo, bio-acumulación, seguridad alimentaria.

\begin{abstract}
Contamination by heavy metals in water resources, soil and air poses one of the most severe problems that compromise food safety and public health at global and local level. In this review, the specific problem of contamination by mercury $(\mathrm{Hg})$, arsenic (As), cadmium ( $\mathrm{Cd}$ ) and lead $(\mathrm{Pb})$ in the environment and food is presented. A description of the sources of contamination, exposure in living beings, accumulation and retention in food and consumer products is carried out. Study cases and results in some countries included Colombia are discussed.
\end{abstract}

Key words: Heavy metals, Mercury, Arsenic, Cadmium, Lead, bio-accumulation, food security.

\section{INTRODUCCIÓN}

La contaminación ambiental se posiciona como uno de los más importantes problemas que afectan a la sociedad del siglo XXI. La pérdida de calidad del aire, del recurso hídrico y de suelos disponibles para actividades agrícolas se ha incrementado exponencialmente (Singh et. al, 2010; Chen et. al, 2013). La tasa de contaminación del agua puede ser estimada en 2000 millones de metros cúbicos diarios. Se hace evidente una crisis de este recurso para los próximos años, lo que podría comprometer el cumplimiento de uno de los objetivos de Desarrollo del Milenio de la Organización de Naciones Unidas (ONU-DAES, 2005-2015). En Septiembre de 2015, la Asamblea general de la ONU, acordó como objetivo: "asegurar la disponibilidad y la gestión sostenible de agua y saneamiento para todos", otorgándole al agua un carácter prioritario para todos los países miembro. Cabe destacar que el año 2015 fue crítico para la agenda en materia de agua y saneamiento.

Cómo citar este artículo:

Reyes, Y.C., Vergara, I., Torres, O.E., Díaz-Lagos, M., \& González, E.E. (2016). Contaminación por metales pesados: Implicaciones en salud, ambiente y seguridad alimentaria. Revista Ingeniería Investigación y Desarrollo, 16 (2), pp. 66-77 
Específicamente, la contaminación del agua por metales pesados ocasionada por vía antrópica y natural, está afectando drásticamente la seguridad alimentaria y salud pública (efsa, 2015; Huang et. al, 2014). Estudios recientes reportan la presencia de metales pesados y metaloides tales como mercurio $(\mathrm{Hg})$, arsénico (As), plomo $(\mathrm{Pb})$, cadmio $(\mathrm{Cd})$, zinc $(\mathrm{Zn})$, níquel (Ni) y cromo $(\mathrm{Cr})$ en hortalizas tales como la lechuga, repollo, calabaza, brócoli y papa (Singh et. al, 2010; Chen et. al, 2013). Esta contaminación, proviene, entre otros causales, del uso para riego de aguas afectadas (Singh et. al, 2010; Fransisca et. al, 2015; Li et. al, 2015). De igual manera, se han encontrado metales en diferentes concentraciones en peces, carnes y leche resultado de la bio-acumulación y movilidad desde el ambiente a las fuentes hídricas (Singh et al, 2010; Li et al, 2015). Algunas especies tales como ostras, mariscos y moluscos acumulan el cadmio proveniente del agua en forma de péptidos ligadores hasta alcanzar valores de concentración entre 100 y $1000 \mu \mathrm{g} / \mathrm{kg}$. En la carne, el pescado y frutas se han reportado valores de concentración entre 1 y $50 \mu \mathrm{g} / \mathrm{kg}$ y

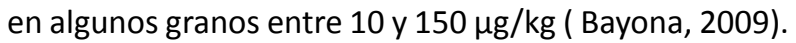

Por su elevada toxicidad, el impacto causado en salud por exposición prolongada o por bio-acumulación de metales pesados resulta alarmante. Dependiendo del tipo de metal o metaloide, se producen afecciones que van desde daños en órganos vitales hasta desarrollos cancerígenos (Combariza, 2009; Nava-Ruíz \& MéndezArmenta 2011). A nivel global, se han reportado casos que dan cuenta de las afecciones en la salud por causa del consumo de alimentos contaminados por metales pesados. Un caso relevante ocurrió en Japón en la década de los cincuenta, en donde la población ubicada en las riberas del río Jintsu, aguas abajo de una zona minera de zinc $(\mathrm{Zn})$, plomo $(\mathrm{Pb})$ y cobre $(\mathrm{Cu})$, se vio afectada por el consumo de arroz proveniente de cultivos contaminados con cadmio (Cd) procedente de los vertimientos de las minas. Esta ingesta produjo una enfermedad conocida como Itai-Itai o osteoartrítis la cual afecta principalmente el tejido óseo (Sánchez et. al., 2010). De otra parte, en la población infantil de Torreón, Coahuila ubicada en Norte-centro de México se han reportado casos por envenenamiento principalmente por plomo $(\mathrm{Pb})$ proveniente de actividades industriales que incorporan este metal a la cadena alimenticia y al agua (Valdés, 1999).

La Organización Mundial de la Salud (OMS) y algunas autoridades ambientales, han establecido niveles de riesgo en función de la concentración de metales en aguas de consumo humano y alimentos. Los mapas realizados en algunas regiones del mundo muestran un importante incremento en la concentración por encima de los límites establecidos, que las clasifica como de alto riesgo (Arnous O.M. et al. 2015; Yuang G.L. et.al, 2014). Así, para el caso del arsénico, la población en riesgo de exposición supera los 150 millones, esto obliga a fortalecer los programas de saneamiento apoyados en tecnologías emergentes como la bio y nanotecnología para el desarrollo de procesos y estrategias experimentales en tareas de detección, cuantificación y remediación (González et.al, 2015).

En la actualidad se acepta de forma generalizada que la distribución, movilidad, disponibilidad biológica y toxicidad de los elementos químicos no es función de la concentración total de los mismos, sino que dependen de la forma química en la que se encuentren (Carusso, J.A. et.al, 2003; Hirose, K. 2006). Es necesario conocer las especies químicas de los elementos para comprender las reacciones químicas y bioquímicas en las que intervienen, y por tanto, obtener información relativa al carácter esencial y tóxico de los elementos químicos. Los análisis de especiación se convertirán en una herramienta esencial para la evaluación de riesgos en el medioambiente, permitiendo que se realicen diagnósticos y controles de los elementos trazas más efectivos. En última instancia, este tipo de investigación puede dar lugar a una legislación ambiental basada en la concentración máxima permisible de especies químicas, en vez de una legislación basada en concentraciones totales de los elementos (Michalke, 2003; Sigg L. et.al, 2006).

\section{EXPOSICIÓN A METALES PESADOS (CD, PB, HG, AS) Y SUS EFECTOS EN LA SALUD Y AMBIENTE}

Como ya fue anotado, la presencia de metales en el ambiente se da por vía natural y antropogénica. Se movilizan en matrices de agua, suelo y aire.

Los metales son persistentes, es decir, no pueden ser creados o degradados, ni mediante procesos biológicos ni antropogénicamente. Una vez que han entrado en los ecosistemas acuáticos, se transforman a través de procesos biogeoquímicos y se distribuyen entre varias especies con distintas características físico-químicas, por ejemplo material particulado $(>0,45 \mu \mathrm{m})$, coloidal $(1 \mathrm{~nm}-0,45 \mu \mathrm{m})$ y especies disueltas $(=1 \mathrm{~nm})$ (Martorell

Rev. I2+D. Vol.16 N². Julio-Diciembre. 2016. Sogamoso-Boyacá, Colombia. 
Reyes, Y.C., Vergara, I., Torres, O.E., Díaz-Lagos, M., \& González, E.E.

J.J., 2010). La materia particulada y coloidal, tanto orgánica como inorgánica, desempeña un papel clave en la coagulación, la sedimentación y en los procesos de adsorción, los cuales influyen en los tiempos de residencia y transporte de los metales trazas desde la columna de agua a los sedimentos y a otras matrices.

La inhalación y la ingesta de alimentos, son dos de las causas más sobresalientes de contaminación. Los efectos tóxicos dependen del tipo de metal, de la concentración y en algunos casos de la edad de la población expuesta. Algunos estudios que evalúan la contaminación de metales pesados en alimentos, carne y leche, han encontrado que el cadmio, el mercurio, el plomo y el arsénico, son cuatro de los elementos que por su impacto en la salud y concentración deben ser cuidadosamente evaluados y monitoreados. La fuente de contaminación y su incorporación en la cadena alimenticia se ilustra en la gráfica de la figura 1.

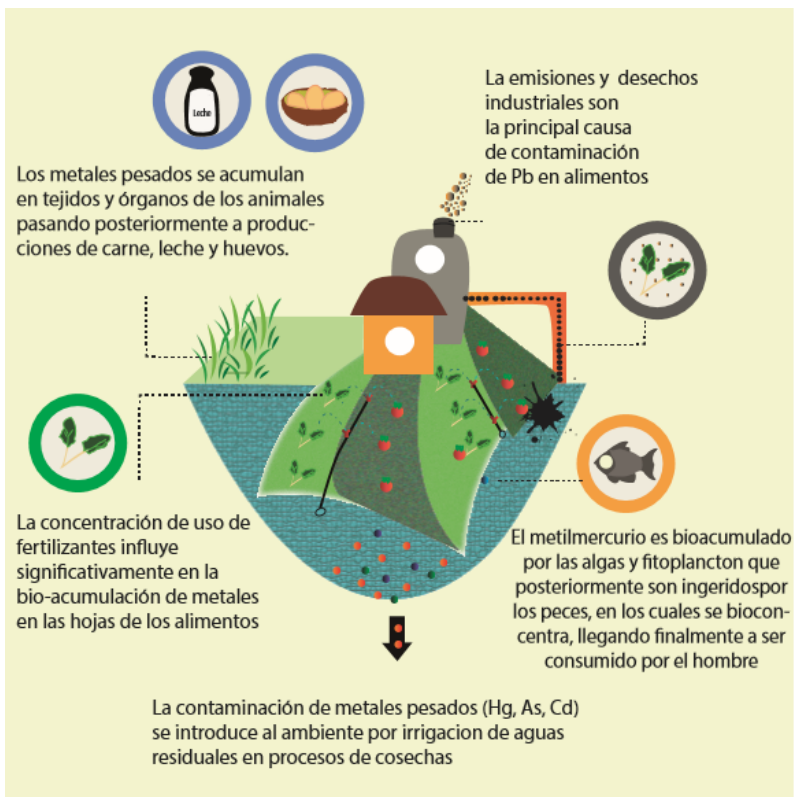

Figura 1. Fuentes de contaminación por metales pesados en aire, suelo, agua y planta. Fuente: El autor.

\subsection{Cadmio $(C d)$}

El cadmio forma parte de la composición natural de algunas rocas y suelos y provoca una liberación al medio ambiente cercana a 25000 toneladas. De otra parte, por vía antrópica las concentraciones en el ambiente pueden ser incrementadas considerablemente. Ya que es un metal ampliamente utilizado en la industria y productos agrícolas, esto ha producido un progresivo aumento en su producción. El $5 \%$ del metal es reciclado y debido a su notable movilidad, provoca una importante contaminación ambiental (Marruecos et.al, 1993).

La población está expuesta al cadmio por diversas vías: i) Oral, a través del agua y la ingesta de comida contaminada con este elemento (hojas de vegetales, granos, cereales, frutas, vísceras animales y pescado) (Nava-Ruíz \& Méndez-Armenta 2011). En algunos países de Europa y Norte América la ingesta diaria de cadmio varía entre 10 y 40 mg/día. ii) La inhalación de partículas durante actividades industriales en personas laboralmente expuestas, donde la concentración de cadmio puede tener valores superiores a $50 \mu \mathrm{g} / \mathrm{L}$. iii) El cigarrillo, cuyo nivel de cadmio está asociado al suelo donde crece el tabaco. Estudios realizados muestran que las concentraciones de cadmio en la sangre para no fumadores varía entre 0,4 a $1,0 \mu \mathrm{g} / \mathrm{L}$, mientras que en fumadores los valores varían entre 1,4 a $4 \mu \mathrm{g} / \mathrm{L}$ (NavarroAviñó et. al, 2007; Bayona, 2009; Nava-Ruíz \& MéndezArmenta 2011). iv) Finalmente, por vía dérmica, aunque las concentraciones absorbidas son muy reducidas.

El cadmio que ingresa por vía respiratoria o por vía oral, se transporta a la sangre y se concentra en el hígado y el riñón. El cadmio tiene la capacidad de acumularse en estos órganos vitales lo que produce daños irreversibles aún para concentraciones reducidas. De otra parte el tiempo de permanencia en estos órganos puede ser muy elevado. Así, el tiempo de vida media del cadmio en el riñón puede alcanzar los 30 años.

Al cadmio se le reconoce como uno de los metales pesados con mayor tendencia a acumularse en las plantas. El cadmio causa severos desequilibrios en los procesos de nutrición y transporte de agua en las plantas (Singh \& Tewari, 2003). La favorabilidad de acumulación de cadmio en las plantas ha llevado a considerarlas como potenciales candidatos para tareas de fitoremediación de este metal.

\subsection{Plomo $(\mathrm{Pb})$}

El plomo es un metal pesado que se ha utilizado durante muchos años debido a su resistencia a la corrosión, ductibilidad, maleabilidad y facilidad para formar aleaciones. El plomo es absorbido por inhalación, ingestión y a través de la piel (Bayona, 2009; NavaRuíz \& Méndez-Armenta 2011). Las principales vías de

Rev. I2+D. Vol.16 N². Julio-Diciembre. 2016. Sogamoso-Boyacá, Colombia. 
exposición son: i) inhalación de partículas de plomo generadas por combustión de algunos materiales. ii) La ingestión de polvo, agua o alimentos contaminados (Zurera et. al, 1987). Tiende a distribuirse en diferentes órganos, tejidos, huesos y dientes, donde se va acumulando con el paso del tiempo (Sanín et. al, 1998). La intoxicación por plomo varía de acuerdo a la edad de la persona y su nivel de exposición (Bayona, 2009).

\subsection{Mercurio $(\mathrm{Hg})$}

Es un metal líquido a temperatura ambiente, que además de encontrarse en su estado elemental, se puede hallar como derivados inorgánicos y derivados orgánicos. El mercurio elemental es poco soluble y por lo tanto poco tóxico al ingerirse, pero puede emitir vapores tóxicos a cualquier temperatura y ocasionar intoxicaciones agudas y crónicas por su inhalación (Sarmiento et. al. 1999; Bayona, 2009).

La toxicidad que exhibe el mercurio depende drásticamente de la fase química en la que se encuentre. El metilmercurio es una de las formas con elevada toxicidad y es muy fácilmente incorporado en la cadena alimenticia y bio-acumulado en seres vivos. Afecta principalmente al sistema nervioso y puede producir graves daños en el cerebro en estado fetal. Es activamente perjudicial para el sistema cardiovascular y puede ser cancerígeno.

\subsection{Arsénico (As)}

Es un elemento ampliamente distribuido en la atmósfera, en la hidrosfera y en la biosfera, el cual está presente en cuatro estados de oxidación As(V), As(III), As(0) y As(-III). Las especies formadas según el estado de oxidación son variadas y pueden ser de origen inorgánico $u$ orgánico. El As(III) puede provenir de la reducción biológica del As (V), y predomina en zonas cercanas a industrias con efluentes ricos en As(III), aguas geotermales y ambientes reductores (Smedley \& Kinniburgh, 2002). En general, en aguas superficiales, el $\mathrm{As}(\mathrm{V})$ predomina sobre el As(III) especie de mayor toxicidad. En aguas subterráneas pueden encontrarse ambos estados de oxidación ya que las concentraciones de $\mathrm{As}(\mathrm{III})$ y $\mathrm{As}(\mathrm{V})$ dependen de la entrada del As al sistema, de las condiciones redox y de la actividad biológica.

En aguas marinas, la especie dominante es el $A s(V)$, que puede ser transformado a formas orgánicas o reducido biológicamente a As(III). El arsénico puede circular en los ecosistemas naturales por un largo periodo de tiempo y puede ser incorporado en suelos, aguas subterráneas y litologías hospedantes (Smedley \& Kinniburgh 2002).

Los efectos toxicológicos del As no son bien conocidos y se especula sobre el proceso de transferencia a los seres humanos (D'Ambrosio, 2005). La arsenicosis o hidroarsenicismo crónico es una enfermedad que se presenta por elevadas concentraciones de As inorgánico y presenta diferentes afectaciones en la salud humana tales como problemas respiratorios, enfermedades cardiovasculares, gastrointestinales y efectos cancerígenos (pulmón, vejiga y piel) entre otras (Marruecos et.al, 1993).

\section{CONTAMINACIÓN POR METALES PESADOS EN ALIMENTOS}

Para el caso de contaminación por metales pesados en alimentos, los límites máximos permisibles en concentración de metales pesados establecidos por la unión europea y la FAO, varían de acuerdo al tipo de alimento (ver tabla 1).

Tabla 1: Límites máximos permisibles de concentración de metales pesados $(\mathrm{Hg}, \mathrm{As}, \mathrm{Cd}$ y $\mathrm{Pb}$ ) en agua, suelo y alimentos de consumo humano. Información obtenida de (Codex, 1995; UE, 2016)

\begin{tabular}{|c|c|c|c|c|c|}
\hline Alimento & Unid. & $\mathrm{Hg}$ & As & $\mathrm{Cd}$ & $\mathrm{Pb}$ \\
\hline $\begin{array}{l}\text { Agua de } \\
\text { consumo } \\
\text { humano }\end{array}$ & \multirow{4}{*}{$\mathrm{mg} / \mathrm{L}$} & 0,001 & 0,05 & 0,01 & 0,05 \\
\hline $\begin{array}{l}\text { Agua Marina y } \\
\text { de estuarios }\end{array}$ & & 0,0001 & 0,05 & 0,05 & 0,01 \\
\hline $\begin{array}{l}\text { Agua de uso } \\
\text { agrícola }\end{array}$ & & 0,001 & 0,1 & 0,01 & 0,05 \\
\hline $\begin{array}{l}\text { Agua para uso } \\
\text { pecuario }\end{array}$ & & 0,01 & 0,2 & 0,05 & 0,05 \\
\hline
\end{tabular}




\begin{tabular}{|c|c|c|c|c|c|}
\hline Alimento & Unid. & $\mathrm{Hg}$ & As & $\mathrm{Cd}$ & $\mathrm{Pb}$ \\
\hline $\begin{array}{l}\text { Hortalizas de } \\
\text { bulbo }\end{array}$ & & nan & nan & 0,05 & 0,1 \\
\hline $\begin{array}{l}\text { Hortalizas } \\
\text { de fruto } \\
\text { (cucurbitáceas) } \\
\end{array}$ & & nan & nan & 0,05 & 0,1 \\
\hline $\begin{array}{l}\text { Hortalizas de } \\
\text { hoja }\end{array}$ & & nan & nan & 0,1 & 0,3 \\
\hline Legumbres & & nan & nan & nan & 0,2 \\
\hline $\begin{array}{l}\text { Raíces y } \\
\text { tubérculos }\end{array}$ & & nan & nan & 0,1 & 0,1 \\
\hline $\begin{array}{l}\text { Carne de } \\
\text { vacuno, porcino } \\
\text { y ovino }\end{array}$ & $\mathrm{mg} / \mathrm{kg}$ & nan & nan & 0,05 & 0,1 \\
\hline Carne de aves & & nan & nan & nan & 0,1 \\
\hline Leche & & nan & nan & nan & 0,02 \\
\hline $\begin{array}{l}\text { Cereales en } \\
\text { grano (excepto } \\
\text { trigo) } \\
\end{array}$ & & nan & 1 & nan & 0,2 \\
\hline peces & & 0,5 & 2 & nan & nan \\
\hline $\begin{array}{l}\text { peces } \\
\text { depredadores }\end{array}$ & & 1 & nan & nan & nan \\
\hline
\end{tabular}

ver drásticamente damnificados en su producción. Esta delicada problemática ha motivado la puesta en marcha de iniciativas orientadas a evaluar los niveles de contaminación por metales pesados en aguas, aire y suelos.

Respecto a la interacción de metales pesados con agroproductos, se han propuesto las siguientes tareas:

i) Evaluar la acumulación de metales pesados sobre las hojas de vegetales expuestas a fertilizantes que contienen grandes cantidades de $\mathrm{Cd}, \mathrm{Pb}, \mathrm{Hg}$ entre otros.

ii) Identificar la toxicidad de metales pesados en hortalizas como brócoli y lechuga.

Los resultados obtenidos muestran que la concentración de metales está relacionada con las condiciones del cultivo. En cultivos expuestos al aire libre se encuentran bajas concentraciones de $\mathrm{As}, \mathrm{Cd}, \mathrm{Cu}, \mathrm{Hg}$ y $\mathrm{Zn}$ pero con elevadas concentraciones de $\mathrm{Pb}$, siendo la deposición atmosférica la principal causa de esta contaminación. Mientras que en cultivos en condiciones de invernadero, las concentraciones son bajas. La presencia de actividades industriales en las vecindades de las zonas de cultivo influye en la presencia las altas concentraciones de $\mathrm{Cu}$ y $\mathrm{Zn}$.

La concentración de metales pesados es mayor en la rizosfera, aunque el uso de fertilizantes influye significativamente en la bio-acumulación de metales en las hojas de los alimentos de suelos contaminados. La concentración de metales es mayor en las hojas que en las raíces de la planta.

\subsection{Contaminación por metales pesados en India}

En India el uso de aguas contaminadas para irrigación es una actividad que se desarrolla con frecuencia en algunas zonas del país. Se han desarrollado estudios que evalúan entre otros aspectos: i) los riesgos en la salud por ingesta de vegetales, cereales y leche; ii) la bio-acumulación de metales en algunas especies como calabaza de botella, calabaza esponja, coliflor, repollo, berenjena, tomates, amaranto, calabaza, rábano, trigo y arroz; iii) el incremento en las concentraciones de metales como el $\mathrm{Cd}, \mathrm{Cu}, \mathrm{Pb}, \mathrm{Zn}, \mathrm{Ni}$ y $\mathrm{Cr}$ en suelo cuando 
se emplea agua de riego contaminada y agua de riego limpia.

Aunque los resultados obtenidos demuestran que los niveles se encuentran por debajo de los límites establecidos, se resalta la importancia de estudios de monitoreo y regulación que garantice la seguridad alimentaria.

\subsection{Contaminación por metales pesados en Grecia}

Estudios realizados en Grecia (Fytianos et.al, 2001) con muestras de vegetales (espinaca, puerro, repollo, lechuga, cebolla, coliflor, apio, remolacha, zanahoria y endivia) tomadas de suelos rurales e industriales mostraron el impacto causado por la actividad industrial en contaminación de estos alimentos por metales pesados. No cabe duda que este es uno de los principales factores que contribuyen a la incorporación de estos contaminantes en aguas, aire y suelos.

En América Latina la gran mayoría de países padecen el problema de contaminación por metales pesados en agua (Bundschuh et. al, 2012). En países como México, Argentina, Chile, el Salvador, Nicaragua, Perú y Bolivia, cerca de cuatro millones de personas consumen aguas contaminadas por arsénico.

\subsection{Contaminación por metales pesados en Colombia}

En las últimas décadas los estudios de calidad de los sistemas acuáticos continentales (ríos, lagos, embalses, etc) han tenido un creciente interés por aspectos como: el incremento de la población en sus riberas, el creciente grado de industrialización, los aportes de los sectores agrícolas, ganaderos y mineros. La importancia que tiene el estudio de metales pesados en diferentes matrices es por la elevada toxicidad, la alta persistencia y rápida acumulación por los organismos vivos, sus efectos no se detectan fácilmente a corto plazo. La toxicidad de estos metales pesados es proporcional a la facilidad de ser absorbidos por los seres vivos.

En Colombia, durante el año 2013, se realizaron 169 muestreos de cadmio, 180 muestreos de cromo y plomo y 104 muestreos de mercurio (IDEAM, 2014). Con respecto al $\mathrm{Hg}$, según se reporta los valores de concentración más altos se encuentran en el río Marmato, Nechí, Magdalena, Guachal y río Coello. Con respecto al Cd las mayores concentraciones se identificaron en río Negro, río Bogotá y río Cararé. En los ríos Marmato, Bogotá, Cauca la Pintada, Achi y Pinillos registraron las concentraciones más altas en $\mathrm{Pb}$.

La cuenca del río Bogotá juega un papel estratégico en la sabana del Centro del país, particularmente en actividades agrícolas, en industriales (Miranda et.al, 2011). La cuenca se divide en tres tramos: cuenca alta, que inicia en el Páramo de Guacheneque y finaliza en Chía. La cuenca media parte de Chía y finaliza en Sibaté. Finalmente la Cuenca baja que desemboca en el río Magdalena. A lo largo de todo el tramo, se presenta contaminación de metales pesados como resultado de la agroindustria y actividad minera (Cuenca alta), curtiembres en la localidad de San Benito (Cuenca Media) e industria ganadera, explotación ganadera e industrial (Cuenca baja).

La cuenca media es la principal fuente de agua para la producción agrícola en los municipios aledaños del noroccidente y sur de la ciudad de Bogotá. Los vertimientos presentan diferentes concentraciones de metales pesados ( $\mathrm{Hg}, \mathrm{As}, \mathrm{cd}$ y $\mathrm{Pb}$ ) como se indica en la tabla 1 y 2. Estos metales afectan la población expuesta que habita en las riveras del río. El agua es empleada para regadío, lo que promueve la movilidad de los metales a suelos y plantas tales como lechugas, repollo y brócoli, entre otros utilizadas para consumo humano (Miranda et.al 2008).

De los trabajos realizados en la cuenca del río Bogotá, cabe destacar el estudio y evaluación del impacto de contaminación sobre la salud en el embalse del Muña, ubicado en Sibaté cerca a Bogotá (Sarmiento et al. 1999; Bayona 2009). La determinación de la concentración de $\mathrm{Hg}, \mathrm{Cd}, \mathrm{Pb}$ y As en cultivos hortícolas de la sabana de Bogotá, presentes en los suelos y en los cultivos de apio, repollo, lechuga y Brócoli, regados con aguas provenientes del río Bogotá, a través del distrito de riego La Ramada (Miranda et.al, 2008). El Desarrollo de estudios socio-demográficos y epidemiológicos de poblaciones expuestas a la contaminación por $\mathrm{Hg}, \mathrm{Cd}$ y $\mathrm{Pb}$ en zonas ribereñas del río Bogotá (Sánchez et.al, 2010). La cuantificación de $\mathrm{As}, \mathrm{Cd}, \mathrm{Hg}, \mathrm{Pb}$ y $\mathrm{Cu}$ en leche cruda y procesada proveniente de hatos lecheros (Madero \& Marrugo, 2011; Franco \& Fernando, 2014; Pinzón, 2015) problemática que se ha reportado igualmente en Antioquia (Franco \& Fernando, 2014). Identificación de la pre-

Rev. I2+D. Vol.16 N². Julio-Diciembre. 2016. Sogamoso-Boyacá, Colombia. 
Reyes, Y.C., Vergara, I., Torres, O.E., Díaz-Lagos, M., \& González, E.E.

sencia de metales en alimentos distribuidos en plazas y supermercados de Bogotá (Méndez et. al, 2007).

Tabla 2. Concentración de As y Hg en muestras de agua del río Bogotá (Reyes, 2015).

Concentración de As $\mu \mathrm{g} / \mathrm{L}$

\begin{tabular}{cc}
\hline Estaciones & As \\
\hline Humedal Jaboque & 0,66 \\
\hline Parque la Florida & 0,42 \\
\hline Cota & 0,43 \\
\hline
\end{tabular}

Tabla 3. Concentración de metales $(\mathrm{Hg}, \mathrm{As}, \mathrm{Cd}, \mathrm{Pb}$ y $\mathrm{Zn})$ en muestras de agua para riego (Mosquera, 2014).

\begin{tabular}{cccccc}
\hline & \multicolumn{6}{c}{ Concentración de metales en $\mu \mathrm{g} / \mathrm{L}$} \\
\hline & $\mathrm{Hg}$ & $\mathrm{As}$ & $\mathrm{Cd}$ & $\mathrm{Pb}$ & $\mathrm{Zn}$ \\
\hline Mosquera & 3 & 4 & 0 & 9 & 12 \\
\hline
\end{tabular}

Estos estudios revelan la necesidad de desarrollar estrategias efectivas de remediación del Río Bogotá desafortunadamente deteriorado por la descarga de desechos domésticos e industriales provenientes de la Sabana de Bogotá y municipios circundantes. El $90 \%$ de la carga contaminante la recibe de los ríos Salitre o Juan Amarillo, Fucha y Tunjuelo.

En otro escenario, el Lago de Tota ubicado a $3015 \mathrm{~m}$ en la zona central de la cordillera oriental de los Andes Colombianos, tiene carácter de humedal y forma parte de los ecosistemas de páramo; su temperatura oscila entre 8 y $15^{\circ} \mathrm{C}$, y posee una belleza natural de gran atractivo turístico.

Tiene forma elongada con eje principal en dirección NE-SW (Figura 2); el área del espejo de agua es de aproximadamente $60 \mathrm{~km}^{2}$ y su cuenca tributaria es de aproximadamente $170 \mathrm{~km}^{2}$, con una profundidad media de 30 m y máxima de 65.5 m (IDEAM, 2014).

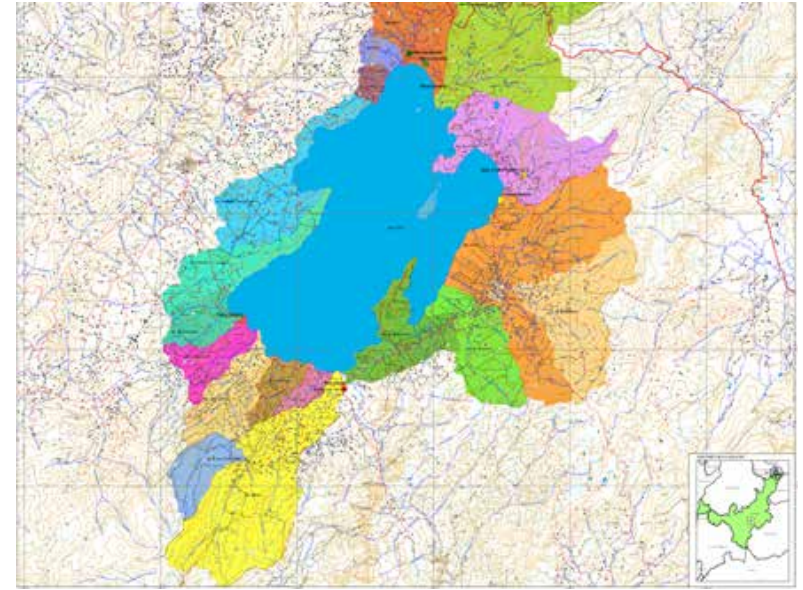

Figura 2. Ilustración del lago de Tota, y su cuenca. Fuente: EI autor

El Lago de Tota es la principal fuente de abastecimiento de agua para consumo humano para cerca de 250.000 habitantes. La extracción del agua se ha incrementado en un 33\% durante el periodo $2000-2010$ y se estima que la demanda crecerá al $81 \%$ en el periodo 2011-2030 (CONPES, 2014).

Las condiciones naturales de clima, altitud, disponibilidad de agua, suelos planos y fértiles que bordean el lago, favorecieron el monocultivo de la cebolla junca (Allium fistulosum Linnaeus), estimulado además por la ventaja de ofrecer varias cosechas al año. El incremento de áreas cultivadas para cebolla fue bastante notable hasta llegar a cubrir más del $90 \%$ del área plana cultivable (CAR, 1978) en menos de dos décadas (Figura 2), en una extensión cercana a $2500 \mathrm{Ha}$.

Las prácticas de cultivo utilizan comúnmente abonos orgánicos, agroquímicos y plaguicidas en gran cantidad (Pesca, 2015) y se ha determinado que la aplicación de fungicidas, pesticidas y fertilizantes presentan sobredosificación en cantidad y frecuencia de aplicación (Torres \& Ramírez, 2014) que incrementa la probabilidad de presencia de metales pesados en suelos agua y alimentos (CONPES, 2014). 

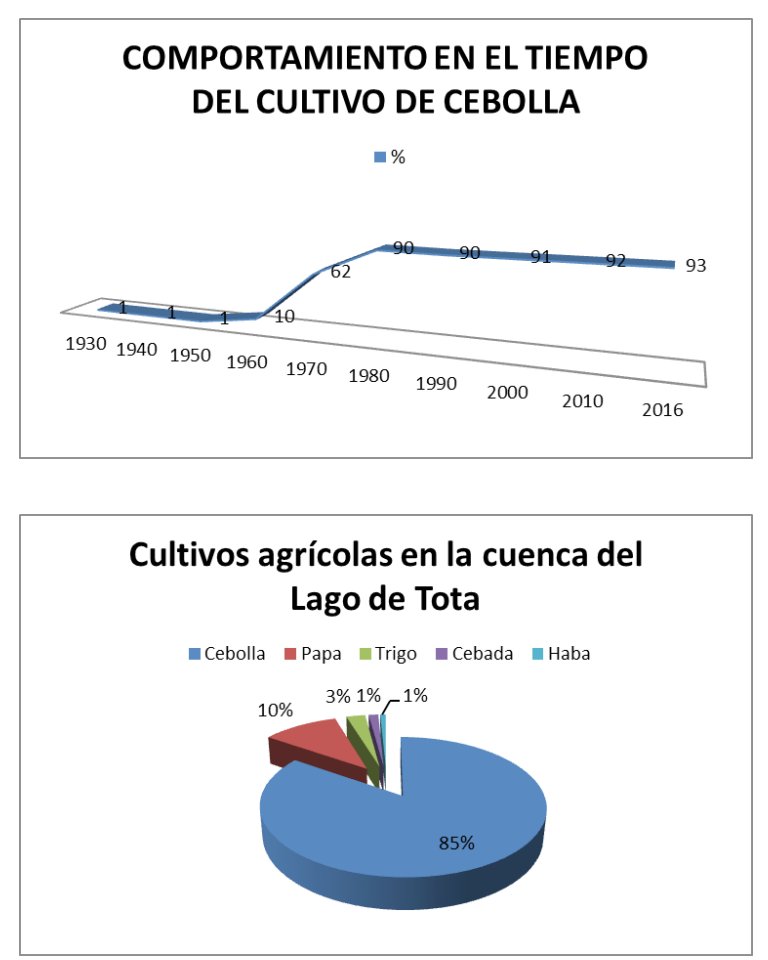

Figura 3. a) La figura ilustra el incremento acelerado del cultivo de cebolla en las década 1960, 1970 y 1980 y b) la comparación del cultivo de cebolla con respecto a otros cultivos en la zona.

Así mismo, la extensión, profundidad del lago, temperatura, demanda y cercanía a centros urbanos, estimularon la producción de trucha arco iris (Oncorhynchus mykiss) mediante cultivo en jaulas dentro del lago; la producción de trucha representada en aproximadamente 100 toneladas al mes (CONPES, 2014), requieren el suministro de alimento para su crianza y generan por consiguiente aportes al lago por excedentes y desechos orgánicos.

En el lago se identifican entre otros problemas ambientales, la contaminación de fuentes hídricas por utilización de agroquímicos, gallinaza, fungicidas y pesticidas en los cultivos, falta de tratamiento de aguas servidas a nivel urbano como municipal e ineficiencia en los sistemas de riego.

En Colombia no son aún suficientes los estudios realizados sobre contaminación por metales pesados. Se hace necesaria la realización de mediciones sistemáticas y continúas bajo estándares de calidad, que permitan construir los mapas de contaminación, necesarios para trazar las estrategias de mitigación y remediación (MADS, 2012).

Aunque no se ha reportado en la literatura estudios que indiquen diferentes concentraciones de metales en el Lago y alimentos de consumo humano, ya se observan iniciativas en ese sentido como la investigación en curso (UPTC, 2016) relacionada con la determinación de metales pesados en la estructura de la Elodea (Egeria Densa); también hay esfuerzos en la búsqueda de soluciones para el tratamiento de aguas residuales de pequeño caudal y prácticas de cultivo orgánico de la cebolla tendientes a reducir el uso de agroquímicos y fungicidas, así como propuestas en redes de sensado para diferentes agroquímicos en el entorno del lago.

Particularmente lo importante de estos estudios es establecer los niveles totales de metales presentes en las diferentes matrices, así como conocer la distribución en las distintas fracciones químicas (especiación) en los diferentes compartimentos medioambientales. Identificar las formas químicas más biodisponibles y en consecuencia con mayor potencial tóxico de los metales estudiados, para la evaluación de los efectos tóxicos debida a la contaminación. Los estudios de laboratorio realizados durante los últimos años, han avanzado los conocimientos de la química de metales en los sistemas acuáticos, incluyendo la formación de complejos metálicos orgánicos e inorgánicos y la adsorción a las partículas de materia orgánica. Investigaciones paralelas han dado lugar a una mejor comprensión de las bases fisiológicas de por qué los metales son tóxicos para los organismos acuáticos. Estos estudios, conducen a una mejora para analizar cómo la química del agua afecta la biodisponibilidad y cómo los metales ejercen toxicidad en el organismo.

Todos estos estudios y acciones, desarrollados en su mayoría de forma aislada, muestran el interés de la comunidad por encontrar soluciones para frenar el deterioro de este valioso cuerpo de agua, y se espera que junto con la voluntad política del gobierno se encausen las acciones necesarias para su protección y den lugar a una legislación ambiental basada en la concentración máxima permisible de especies químicas, en vez de una legislación basada en concentraciones totales de los elementos.

Rev. I2+D. Vol.16 N². Julio-Diciembre. 2016. Sogamoso-Boyacá, Colombia. 
Reyes, Y.C., Vergara, I., Torres, O.E., Díaz-Lagos, M., \& González, E.E.

\section{CONCLUSIONES}

A nivel global y local se identifica un creciente problema de contaminación por metales pesados, que compromete severamente la salud, seguridad alimentaria y medio ambiente.

Los estudios demuestran que la leche de bovinos que pastorean e ingieren agua, pastos o forrajes contaminados por metales pesados ( $\mathrm{Hg}, \mathrm{As}, \mathrm{Cd}$ y $\mathrm{Pb}$ ) influye sobre las concentraciones de dichos elementos en la leche y carne. De igual manera las condiciones de cultivo influyen en la concentración de metales pesados sobre las diferentes matrices (aire, agua, suelo y plantas).

Los límites máximos permisibles de concentración de metales está muy bien establecido en agua. Sin embargo aún falta por definir los límites de concentración y riesgo de $\mathrm{Hg}$, As y $\mathrm{Cd}$ en hortalizas, legumbres y cereales, para permitir estandarizar y consensuar los estudios de contaminación que se están realizando, así como los efectos tóxicos y en ambiente.

Se recomienda realizar monitoreo y detección de contaminantes en agua, fauna y flora, para trazar mapas de concentración de estos contaminantes y favorecer los planes de acción conducentes a tareas de mitigación y remediación. Es importante atender puntos estratégicos de Colombia, como el rio Bogotá y el Lago de Tota, entre otros.

Es necesario realizar estudios sistemáticos, científicos y complementarios del contenido de metales pesados en diferentes matrices que permitan la elaboración de informes fehacientes y con visión del futuro sobre el estado del rio Bogotá y el Lago de Tota e incidir directamente sobre la calidad y la localización, con ello se puede facilitar una mejor identificación y control de dichas afluencias para que los organismos pertinentes puedan tomar medidas preventivas y decisiones de mejora. El análisis de especiación proporciona información respecto a la biodisponibilidad en unas determinadas condiciones medioambientales y pequeños cambios en los parámetros fisicoquímicos del medio.

\section{REFERENCIAS}

Arnous O.M, Hassan A.A.M. (2015). Heavy metals risk assessment in water and bottom sediments of the eastern part of Lake Manzala, Egypt, based on remote sensing and GIS. Arabian Journal of Geosciences, 8, (10), pp. 7899-7918. DOI: http://dx.doi.org/10.1007/s12517-014-1763-6

Carusso, J.A, Klaue, B. Michalke, B, Rocke, D.M. 2003. Group assessment: elemental speciation. Ecotox. Environ. Safe, 56, 32-44. DOI: http://dx.doi. org/10.1016/S0147-6513(03)00048-4

Combariza, B.D.A. 2009. Contaminacion por Metales Pesados en el embalse del Muña y su relación con los niveles en sangre de Plomo, Mercurio y Cadmio y alteraciòn de salud enlos habitantes del municipio de Sibaté (Cundinamarca) pp.1115. Trabajo de Maestria, Universidad Nacional de Colombia.

Bundschuh, J. et al., 2012. One century of arsenic exposure in Latin America: A review of history and occurrence from 14 countries. Science of the Total Environment.

Chen, Y. et al., 2013. Accumulation and health risk of heavy metals in vegetables from harmless and organic vegetable production systems of China. Ecotoxicology and Environmental Safety. DOI: http://dx.doi.org/10.1016/j. ecoenv.2013.09.037

Codex Stand . 1995. Norma general del codex para los contaminantes y las tóxinas presentes en los alimentos. Recuperado de: http://www.fao. org/fileadmin/user_upload/livestockgov/documents/CXS_193s.pdf

Colombia, Ministerio de Ambiente y Desarrollo Sostenible. (2012). Diagnóstico Nacional de Salud Ambiental. Recuperado de: https://www.minsalud.gov.co/sites/rid/Lists/BibliotecaDigital/ RIDE/INEC/IGUB/ diagnostico\%20de\%2 0salud\%20Ambiental\%20compilado.pdf 
Consejo Nacional de Política Económica y Social Conpes. (2014). Manejo Ambiental Integral de la Cuenca Hidrográfica del Lago de Tota. Bogotá.

D’Ambrosio, M.C., 2005. Arsénico en aguas: origen, movilidad y tratamiento. Evaluación y selección de tecnologías disponibles para remoción de arsénico, pp.123-136.

Departamento de Asuntos Económicos y Sociales de Naciones Unidas (ONU-DAES). 2005-2015. Recuperado de: http://www.un.org/spanish/waterforlifedecade/.

European Food Safety Authority (efsa).2015. Recuperado de: http://www.efsa.europa.eu/en/topics/ topic/metals

Franco, L., \& Fernando, L. (2014). Presencia de metales pesados en hatos lecheros de los municipios de San Pedro y Entrerríos, Antioquia, Colombia.

Fransisca, Y. et al., 2015. Assessment of arsenic in Australian grown and imported rice varieties on sale in Australia and potential links with irrigation practises and soil geochemistry. Chemosphere, 138, pp.1008-1013. Available at: DOI: http://dx.doi.org/10.1016/j.chemosphere.2014.12.048

Fytianos, K., Katsianis, G., Triantafyllou, P., \& Zachariadis, G. (2001). Accumulation of heavy metals in vegetables grown in an industrial area in relation to soil. Bulletin of environmental contamination and toxicology, 67(3), 0423-0430. DOI: http://dx.doi.org/10.1007/s001280141

González, E., Marrugo, J., \& Martínez, V., (2015). El problema de Contaminación por Mercurio. Nanotecnología: Retos y Posibilidades para Medición y Remediación. Red Colombiana de Nanociencia y Nanotecnología.

Hirose, K. (2006). Chemical speciation of trace metals in seawater. A review .Anal. Sci., 22, 10551063. DOI: http://dx.doi.org/10.2116/analsci.22.1055
Huang, Z. et al., (2014). Heavy metals in vegetables and the health risk to population in Zhejiang, China. Food Control, 36(1), pp.248-252. Available at: DOI: http://dx.doi.org/10.1016/j.foodcont.2013.08.036.

IDEAM (2014). Informe batimetría Lago de Tota. Grupo de Modelación, Subdirección de Hidrología.40p.

IDEAM,Estudio Nacional del Agua. (2014). Recuperado de: http://documentacion.ideam.gov.co /openbiblio/bvirtual/023080/ENA_2014.pdf

Li, N. et al., (2015). Concentration and transportation of heavy metals in vegetables and risk assessment of human exposure to bioaccessible heavy metals in soil near a waste-incinerator site, South China. Science of the Total Environment, 521-522, pp.144-151. Available at: DOI: http:// dx.doi.org/10.1016/j.scitotenv.2015.03.081.

Madero, A., \& Marrugo, J. (2011). Detección de metales pesados en bovinos, en los valles de los ríos Sinú y San Jorge, Departamento de Cordoba, Colombia. Revista MVZ Córdoba, 16(1), 23912401.

Marruecos, L., Nogué, S., \& Nolla, J. (1993). Toxicología clínica. Barcelona.: Springer-Verlag Ibérica.

Martorell J.J. (2010) Biodisponibilidad de metales pesados en dos ecosistemas acuáticos de la costa Suratlántica andaluza afectados por Contaminación difusa. Tesis Doctoral Universidad de Cádiz.

Méndez Fajardo, S. et al., (2007). Estudio preliminar de los niveles de cadmio en arroz, fríjoles y lentejas distribuidos en supermercados de Bogotá y plazas de Manizales. Fitotecnia Colombiana, 7(2), pp.40-47.

Miranda, D., Carranza, C., Rojas, C. A., Jerez, C. M., Fischer, G., \& Zurita, J. (2011). Acumulación de metales pesados en suelo y plantas de cuatro cultivos hortícolas, regados con agua del río Bogotá. Revista Colombiana de Ciencias 
Reyes, Y.C., Vergara, I., Torres, O.E., Díaz-Lagos, M., \& González, E.E.

Hortícolas, 2(2), 180-191. DOI: http://dx.doi. org/10.17584/rcch.2008v2i2.1186

Michalke, B., (2003). Element speciation definitions, analytical methodology and some examples. Ecotox. Environ. Safe., 56, 122-139. DOI: http:// dx.doi.org/10.1016/S0147-6513(03)00056-3

Mosquera, N. (2014). Funcionalización de superficies nano estructuradas para asistir procesos de detección de contaminantes en agua. Ms.C. Thesis, Pontificia Universidad Javeriana.

Navarro-Aviñó, J.P., Aguilar Alonso, I. \& López-Moya, J.R., (2007). Aspectos bioquímicos y genéticos de la tolerancia y acumulación de metales pesados en plantas. Ecosistemas, 16(2), pp.10-25.

Nava-Ruíz, C. \& Méndez-Armenta, M., (2011). Efectos neurotóxicos de metales pesados (cadmio, plomo, arsénico y talio). Archivos de Neurociencias, 16(3), pp.140-147.

Pesca, S.Y. (2015). Seguimiento del comportamiento físico y químico de los agroquìmicos mas utilizados en el cultivo de cebolla larga en el entorno cercano del lago de Tota y aplicación del modelo CEPIS para evaluar su impacto, Trabajo de Grado. Universidad Pedagógica y Tecnológica de Colombia.

Pinzón Choque, C. G. (2015). Determinación de los niveles de plomo y cadmio en leche procesada en la ciudad de Bogotá D.C. Tesis de grado. Universidad Nacional de Colombia. Sede Bogotá

Reyes, Y. (2015). Sistema portable de pretratamiento para asistir nanosensores con capacidad de detección y cuantificación In situ de muestras de agua. Ms.C. Thesis, Pontificia Universidad Javeriana.

Rodríguez-Lado, L., Sun, G., Berg, M., Zhang, Q., Xue, H., Zheng, Q., \& Johnson, C. A. (2013). Groundwater arsenic contamination throughout China.Science (New York, N.Y.), 341(6148), 8668. Retrieved from http://www.ncbi.nlm.nih. gov/pubmed/23970694. DOI: http://dx.doi. org/10.1126/science.1237484
Sánchez, C.I. et al., (2010). Perfil sociodemográfico y epidemiológico de la población expuesta a la contaminación por mercurio, plomo y cadmio, ubicada en la vereda Manuel Sur del municipio de Ricaurte y los barrios Brisas del Bogotá y La Victoria del municipio de Girardot. Investig. Enferm, 12(2), pp.93-116.

Sanín, L. et al., (1998). Acumulación de plomo en hueso y sus efectos en la salud. Salud Pública de México, 40(4). Available at: http://www.redalyc.org/ articulo.oa?id=10640409. DOI: http://dx.doi. org/10.1590/S0036-36341998000400009

Sarmiento, M.I. et al., (1999). Evaluación del Impacto de la Contaminación del Embalse del Muña Sobre la Salud Humana. Revista De Salud Publica, 1(2) (February), pp.159-171.

Sigg, L., Black, F., Buffle, J., Cao, J., Cleven, R., Davidson, W., Galceran, J., Gunkel, P., Kalis, E., Kistler, D., Martin, M., Nol, S., Nur, Y., Odzak, N., Puy, J., van Rimsdijk, W., Temminghoff, E.,Tercier Waeber, M.L., Toepperwien, S., Town, R.M., Unsworth, E., Warnken, K.W., Weng, L., Xue,H., Zhang, H., (2006). Comparasion of analytical techniques for dynamic trace metal speciation in natural freshwaters. Environ. Sci. Technol., 40, 1934-1941. DOI: http://dx.doi.org/10.1021/ es051245k

Singh, P. K., \& Tewari, R. K. (2003). Cadmium toxicity induced changes in plant water relations and oxidative metabolism of Brassica juncea L. plants. Journal of Environmental Biology, 24(1), 107112

Singh, A. et al., (2010). Risk assessment of heavy metal toxicity through contaminated vegetables from waste water irrigated area of Varanasi, India. Tropical Ecology, 51(2 SUPPL.), pp.375-387. Available at: DOI: http://dx.doi.org/10.1016/j. fct.2009.11.041.

Smedley, P.L. \& Kinniburgh, D.G.,(2002). A review of the source, behaviour and distribution of arsenic in natural waters. Applied Geochemistry, 17(5), pp.517-568. DOI: http://dx.doi.org/10.1016/ S0883-2927(02)00018-5 
Torres, B.N \& Ramírez A. L.N. (2014). Caracterizaciòn de la zona de influencia del cultivo de cebolla larga y la determinación de tasas de usos de agroquímicos en el Lago de Tota. Trabajo de Grado. Universidad Pedagógica y Tecnológica de Colombia.

Unión Europea. (2016). Contenidos máximos en métales pesados en productos alimenticios. Recuperado de: http://plaguicidas.comercio.es/MetalPesa.pdf

UPTC (2016). Grupo de Investigación Ingeniería Geológica, Proyecto de Investigación.

Valdés, F., (1999). La contaminacion por metales pesados en Torreón, Coahuila, México. Texas Center for Policy Studies, Primera ed, p.50. Available at: http://www.texascenter.org/publications/ torreon.pdf.

Yuan G. L., Sun T.H., Han P., Li J., Lang X.X. (2014). Source identification and ecological risk assessment of heavy metals in topsoil using environmental geochemical mapping: Typical urban renewal area in Beijing, China. Journal of Geochemical Exploration, 136, pp 40-47. DOI: http://dx.doi. org/10.1016/j.gexplo.2013.10.002

Zurera, G. et al., (1987). Lead and cadmium contamination levels in edible vegetables. Bulletin of Environmental Contamination and Toxicology, 38(5), pp.805-812. DOI: http://dx.doi. org/10.1007/BF01616705 